\title{
Diffractive dijet photoproduction at the EIC
}

\author{
Vadim Guzey $^{a}$ and Michael Klasen ${ }^{b}$ \\ "National Research Center "Kurchatov Institute", \\ Petersburg Nuclear Physics Institute (PNPI), Gatchina, 188300, Russia \\ ${ }^{b}$ Institut für Theoretische Physik, Westfälische Wilhelms-Universität Münster, \\ Wilhelm-Klemm-Straße 9, 48149 Münster, Germany \\ E-mail: guzey_va@nrcki.pnpi.ru, michael.klasen@uni-muenster.de
}

ABSTRACT: We present a first, detailed study of diffractive dijet photoproduction at the recently approved electron-ion collider (EIC) at BNL. Apart from establishing the kinematic reaches for various beam types, energies and kinematic cuts, we make precise predictions at next-to-leading order (NLO) of $\mathrm{QCD}$ in the most important kinematic variables. We show that the EIC will provide new and more precise information on the diffractive parton density functions (PDFs) in the pomeron than previously obtained at HERA, illuminate the still disputed mechanism of global vs. only resolved-photon factorization breaking, and provide access to a completely new quantity, i.e. nuclear diffractive PDFs.

Keywords: Heavy Ion Phenomenology, NLO Computations

ARXIV EPRINT: 2004.06972 


\section{Contents}

1 Introduction 1

2 Analytical approach 3

3 Diffraction on protons 4

3.1 NLO QCD predictions for the EIC 5

$3.2 K$-factors 6

3.3 Evolution of gluon in pomeron contribution $\quad 7$

3.4 Dependence on diffractive PDFs 8

$\begin{array}{lr}3.5 & \text { Range in } x_{\mathbb{P}} \text { and reggeon contribution }\end{array}$

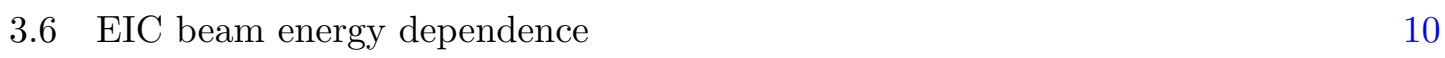

$\begin{array}{lll}3.7 & \text { Factorization breaking } & 11\end{array}$

4 Diffraction on heavy nuclei $\quad 14$

$\begin{array}{lll}4.1 & \text { Nuclear diffractive PDFs and nuclear shadowing } & 14\end{array}$

$\begin{array}{lll}4.2 & \text { NLO QCD predictions for the EIC } & 15\end{array}$

$\begin{array}{lll}4.3 & \text { Factorization breaking } & 15\end{array}$

5 Conclusion $\quad 16$

\section{Introduction}

From 1992 to 2007, hadron-electron collisions at DESY's circular installation HERA provided a wealth of data and information on the strong interaction and the partonic structure of the proton, not only in deep-inelastic scattering (DIS), where the virtuality of the exchanged photon $Q^{2} \gg 1 \mathrm{GeV}^{2}$ is large, but also in photoproduction, where $Q^{2} \leq 1 \mathrm{GeV}^{2}$. While electrons (or positrons) of energy $27.5 \mathrm{GeV}$ mostly collided with protons of first 820 , then $920 \mathrm{GeV}$, the last months of operation were dedicated to lower proton beam energies of 575 and $460 \mathrm{GeV}$ in view of better access to the longitudinal structure function $F_{L}$ and therefore the gluon dynamics at small momentum fractions $x$. While inclusive DIS precisely pinned down the quark parton distribution functions (PDFs), jets (which for dijet invariant masses larger than $16 \mathrm{GeV}$ make up $10-20 \%$ of the inclusive DIS cross section) and photoproduction provided additional constraints on the running of the QCD coupling constant $\alpha_{s}$ and the gluon PDF in the proton $[1,2]$.

The two general purpose detectors H1 and ZEUS were supplemented with forward taggers to identify diffractive processes $e p \rightarrow e X Y$, which — rather surprisingly — accounted for a substantial fraction $(10-15 \%)$ of all events. In DIS, where QCD factorization was proven to hold [3], they could be interpreted in terms of diffractive structure functions. 
Under the additional assumption of Regge factorization [4], the flux of pomeron $(\mathbb{P})$ and higher Regge trajectories like the reggeon $(\mathbb{R})$ can be parametrized, pomeron PDFs could be extracted [5-7] and their universality tested in other types of collisions. It turned out that in hadron-hadron collisions at the Tevatron and the LHC, factorization was broken by a factor of $0.1[8,9]$ to 0.025 [10], depending on the collision energy, the order of perturbative QCD calculations and simplifying assumptions about the non-diffractive structure function [11] and in qualitative agreement with calculations based on multi-pomeron exchanges in a two-channel eikonal model $[12,13]$.

For dijet photoprodution, these calculations suggest that factorization should hold for the DIS-like direct-photon processes, but be broken by a factor of 0.34 for the resolvedphoton processes, where the photon fluctuates before the hard interaction into $q \bar{q}$ pairs and their vector-meson dominated (VMD) bound states [14]. It is, however, well-known that at next-to-leading order (NLO) of QCD [15-18] and beyond [19, 20] direct and resolved processes are connected through the factorization of collinear initial-state singularities, which to preserve factorization-scale independence should also be suppressed [21]. Also, the H1 [22] and ZEUS [23] data can be described by not only suppressing the resolved (and direct initial-state) contribution, but also by a global suppression factor of 0.42 to 0.71 , even though this factor then depends on the transverse jet momentum and is subject to large theoretical uncertainties from scale variations and hadronization corrections [24]. It is also possible that the resolved-photon suppression factor depends on the parton flavor [25]. Elucidating the mechanism of factorization breaking in dijet photoproduction is therefore one of the important desiderata of the HERA program.

Since there is currently no electron-hadron collider in operation, new experimental information can in the short term only be obtained from ultraperipheral collisions (UPCs) at the LHC [26], which have contributions from both photoproduction [27] and diffraction [28]. Dijet photoproduction at the LHC might even provide novel constraints on nuclear PDFs [29] or first information on the yet unknown diffractive nuclear PDFs [28, 30]. In the medium term, the recently approved electron-ion collider (EIC) at BNL [31] has the potential for detailed studies of jets in DIS [32-38] and photoproduction [39-42] in the clean environment of an electron-nucleus collider, which was planned for Run 3 at HERA, but never implemented.

In this paper, we explore in detail the EIC potential for diffractive dijet photoproduction. We begin by reviewing in section 2 our analytical approach based on the factorization of hadronic and partonic cross sections, the extraction of diffractive PDFs from HERA, our NLO QCD calculation of the partonic dijet cross section, and theoretical models for factorization breaking. Section 3 contains a large variety of results for diffraction on protons, starting with NLO QCD predictions for the EIC with colliding beams of $21 \mathrm{GeV}$ electrons and $100 \mathrm{GeV}$ protons and the corresponding $K$ factors as well as studies of the scale evolution of the pomeron PDFs, the range of cross sections predicted from different HERA fits of the diffractive PDFs, of the cross section with a larger range in the longitudinal pomeron momentum fraction $x_{\mathbb{P}}$ and of the corresponding increase of the sub-leading reggeon contribution. We then demonstrate the advantage of a higher proton beam energy of $275 \mathrm{GeV}$ and conclude this section with numerical predictions based on the different approaches to 

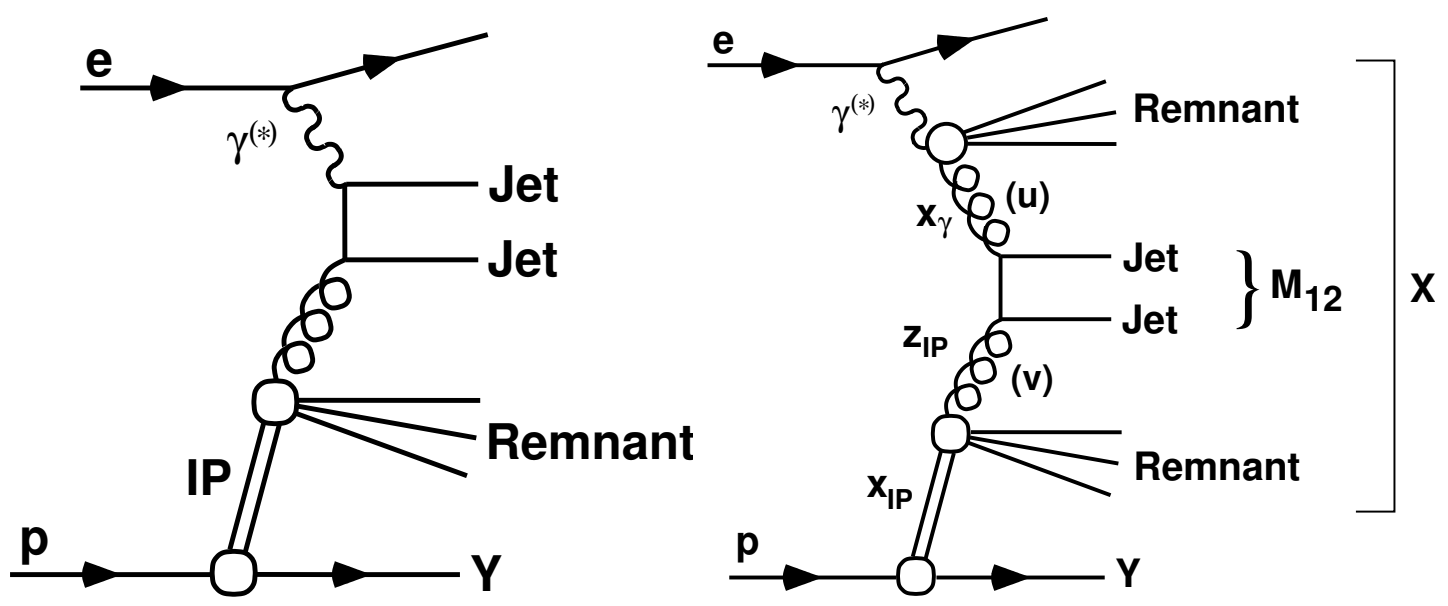

Figure 1. Diffractive production of dijets with invariant mass $M_{12}$ in direct (left) and resolved (right) photon-pomeron collisions, leading to the production of one or two additional remnant jets. The hadronic systems $X$ and $Y$ are separated by the largest rapidity gap in the final state.

factorization breaking. In Section 4 we address the diffraction on nuclei. We start by reviewing the theoretical definition of nuclear diffractive PDFs and the leading-twist model of nuclear shadowing, before we make numerical predictions at NLO for diffractive dijet photoproduction on various nuclei and discuss again the different approaches to factorization breaking. Our conclusions are given in section 5 .

\section{Analytical approach}

At the EIC, like at HERA, electrons $e$ of four-momentum $k$ will collide with protons $p$ of four-momentum $P$ at a squared center-of-mass system (CMS) energy $S=(k+P)^{2}$. For nuclei, the relevant quantity is the squared CMS energy per nucleon and is typically (i.e. for heavy nuclei) smaller by about a factor of $Z / A \approx 0.4$, where $Z$ is the nucleus charge and $A$ is the number of nucleons. In photoproduction, the virtuality $Q^{2}=-q^{2}=-\left(k-k^{\prime}\right)^{2}$ of the radiated photon $\gamma$ is small (typically less than $Q_{\max }^{2}=0.01-1 \mathrm{GeV}^{2}$ ), and its spectrum can be described in the improved Weizsäcker-Williams approximation [43]

$$
f_{\gamma / e}(y)=\frac{\alpha}{2 \pi}\left[\frac{1+(1-y)^{2}}{y} \ln \frac{Q_{\max }^{2}(1-y)}{m_{e}^{2} y^{2}}+2 m_{e}^{2} y\left(\frac{1-y}{m_{e}^{2} y^{2}}-\frac{1}{Q_{\max }^{2}}\right)\right] .
$$

Here, $\alpha$ is the electromagnetic fine structure constant, $k^{\prime}$ is the four-momentum of the scattered electron, $y=(q P) /(k P)$ is its longitudinal momentum transfer and $m_{e}$ its mass.

Diffractive processes are characterized by the presence of a large rapidity gap between the central hadronic system $X$ and the forward-going hadronic system $Y$ with fourmomentum $p_{Y}$, low mass $M_{Y}$ (typically a proton that remained intact or a proton plus low-lying nucleon resonances), small four-momentum transfer $t=\left(P-p_{Y}\right)^{2}$, and small longitudinal momentum transfer $x_{\mathbb{P}}=q\left(P-p_{Y}\right) /(q P)$ (see figure 1).

In dijet photoproduction, the system $X$ contains (at least) two hard jets with transverse momenta $p_{T 1,2}$, rapidities $\eta_{1,2}$ and invariant mass $M_{12}$, as well as remnant jets from 
the diffractive exchange, dominated by the pomeron $\mathbb{P}$ as the lowest-lying Regge trajectory, and from the photon, when the latter does not interact directly with the proton or nucleus, but first resolves into its partonic constituents. Assuming both QCD and Regge factorization, the cross section for the reaction $e+p \rightarrow e+2$ jets $+X^{\prime}+Y$ can then be calculated through

$$
d \sigma=\sum_{a, b} \int d y \int d x_{\gamma} \int d t \int d x_{\mathbb{P}} \int d z_{\mathbb{P}} f_{\gamma / e}(y) f_{a / \gamma}\left(x_{\gamma}, M_{\gamma}^{2}\right) f_{\mathbb{P} / p}\left(x_{\mathbb{P}}, t\right) f_{b / \mathbb{P}}\left(z_{\mathbb{P}}, M_{\mathbb{P}}^{2}\right) d \hat{\sigma}_{a b}^{(n)} .
$$

The $x_{\mathbb{P}}$ dependence is parameterized using a flux factor motivated by Regge theory,

$$
f_{\mathbb{P} / p}\left(x_{\mathbb{P}}, t\right)=A_{\mathbb{P}} \cdot \frac{e^{B_{\mathbb{P}} t}}{x_{\mathbb{P}}^{2 \alpha_{\mathbb{P}}(t)-1}},
$$

where the pomeron trajectory is assumed to be linear, $\alpha_{\mathbb{P}}(t)=\alpha_{\mathbb{P}}(0)+\alpha_{\mathbb{P}}^{\prime} t$, and the parameters $B_{\mathbb{P}}$ and $\alpha_{\mathbb{P}}^{\prime}$ and their uncertainties are obtained from fits to H1 diffractive DIS data [5]. The longitudinal momentum fractions of the parton $a$ in the photon $x_{\gamma}$ and of the parton $b$ in the pomeron $z_{\mathbb{P}}$ can be experimentally determined from the two observed leading jets through

$$
x_{\gamma}^{\mathrm{obs}}=\frac{p_{T 1} e^{-\eta_{1}}+p_{T 2} e^{-\eta_{2}}}{2 y E_{e}} \text { and } z_{\mathbb{P}}^{\mathrm{obs}}=\frac{p_{T 1} e^{\eta_{1}}+p_{T 2} e^{\eta_{2}}}{2 x_{\mathbb{P}} E_{p}} .
$$

$M_{\gamma}$ and $M_{\mathbb{P}}$ are the factorization scales at the respective vertices, and $d \hat{\sigma}_{a b}^{(n)}$ is the cross section for the production of an $n$-parton final state from two initial partons $a$ and $b$. It is calculated in NLO in $\alpha_{s}(\mu)$ [15-18], as are the PDFs of the photon and the pomeron. For the former, we use the GRV NLO parametrization, which we transform from the DIS $\gamma$ to the $\overline{\mathrm{MS}}$ scheme [44]. Our default choice for the diffractive PDFs is H1 2006 Fit B [5], which includes proton dissociation up to masses of $M_{Y}<1.6 \mathrm{GeV}$ and is integrated up to $|t|<1 \mathrm{GeV}^{2}$ and $x_{\mathbb{P}}<0.03$. We identify the factorization scales $M_{\gamma}, M_{\mathbb{P}}$ and the renormalization scale $\mu$ with the average transverse momentum $\bar{p}_{T}=\left(p_{T 1}+p_{T 2}\right) / 2$ [24].

\section{Diffraction on protons}

In this first numerical section, we focus on electron-proton collisions at the EIC with an electron beam energy of $E_{e}=21 \mathrm{GeV}$ and a proton beam energy of $E_{p}=100 \mathrm{GeV}$, which will in the next section also be used as the beam energy per nucleon for electronnucleus collisions. We assume detectors that have the same kinematic acceptance as H1 for diffractive events, i.e. the capability to identify a large rapidity gap and/or a leading proton in a Roman pot spectrometer. We also allow for proton dissociation up to masses of $M_{Y}<$ 1.6 GeV, a four-momentum transfer of $|t|<1 \mathrm{GeV}^{2}$ and a longitudinal momentum transfer of $x_{\mathbb{P}}<0.03$. Photoproduction events are assumed to be selected with (anti-)tagged electrons and photon virtualities up to $Q^{2}<0.1 \mathrm{GeV}^{2}$, assuming full kinematic coverage of the longitudinal momentum transfer $0<y<1$ from the electron to the photon.

Jets are defined with the anti- $k_{T}$ algorithm and a distance parameter $R=1$, where at NLO jets contain at most two partons [45]. Given the limited EIC energy and experience from HERA, we assume that the detectors can identify jets above relatively low tranverse 

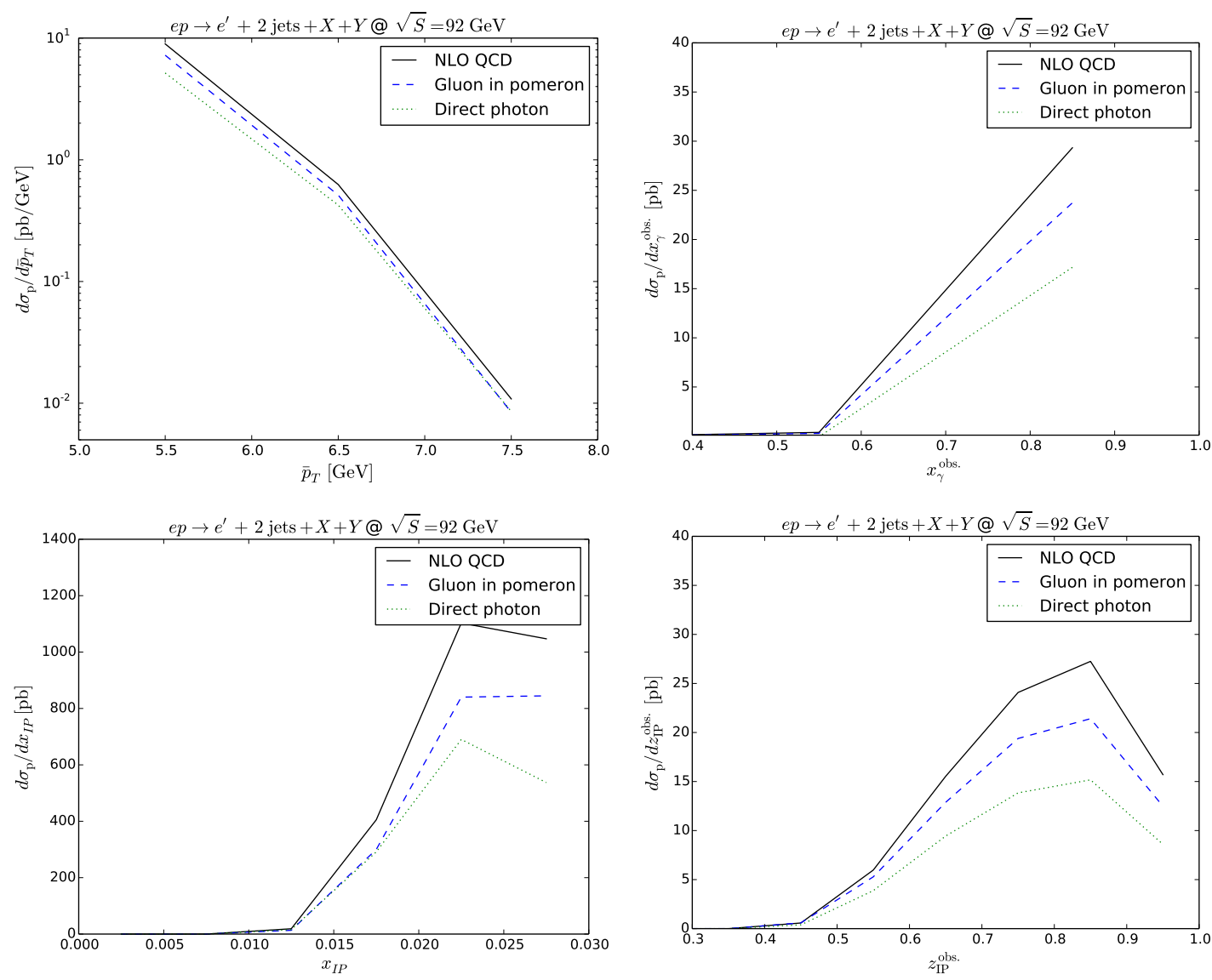

Figure 2. NLO QCD cross sections for diffractive dijet photoproduction at the EIC in our default set-up. Shown are distributions in the jet average transverse momentum (top left) as well as the (observed) longitudinal momentum fractions of the photon (top right), the pomeron (bottom left) and the partons in the pomeron (bottom right). In addition to the total cross section (full black) we also show the contributions from gluons in the pomeron (dashed blue) and direct photons (dotted green curves).

energies of $p_{T 1}>5 \mathrm{GeV}$ (leading jet) and $p_{T 2}>4.5 \mathrm{GeV}$ (subleading jet). This will, however, require a good resolution of the hadronic jet energy scale and subtraction of the underlying event. The latter will also be important to avoid large hadronization corrections of the partons, which are particularly prominent at large $x_{\gamma}$ and have so far obscured the interpretation of the observed factorization breaking. Note also that asymmetric jet $p_{T}$ cuts allow one to avoid an enhanced sensitivity to soft radiation [46]. Rapidities are a priori accepted in the range $\eta_{1,2} \in[-4 ; 4]$. We find, however, that in diffractive photoproduction most jets are central and have an average rapidity $\bar{\eta}=\left(\eta_{1}+\eta_{2}\right) / 2 \in[-1.5 ; 0]$. This range is enlarged to $[-1.5 ; 1]$ at higher proton beam energy or for a larger range in $x_{\mathbb{P}}$, see below.

\subsection{NLO QCD predictions for the EIC}

In figure 1 we show our NLO QCD cross sections for diffractive dijet photoproduction at the EIC in this default set-up, i.e. at a CMS energy of $92 \mathrm{GeV}$. The distribution in the 

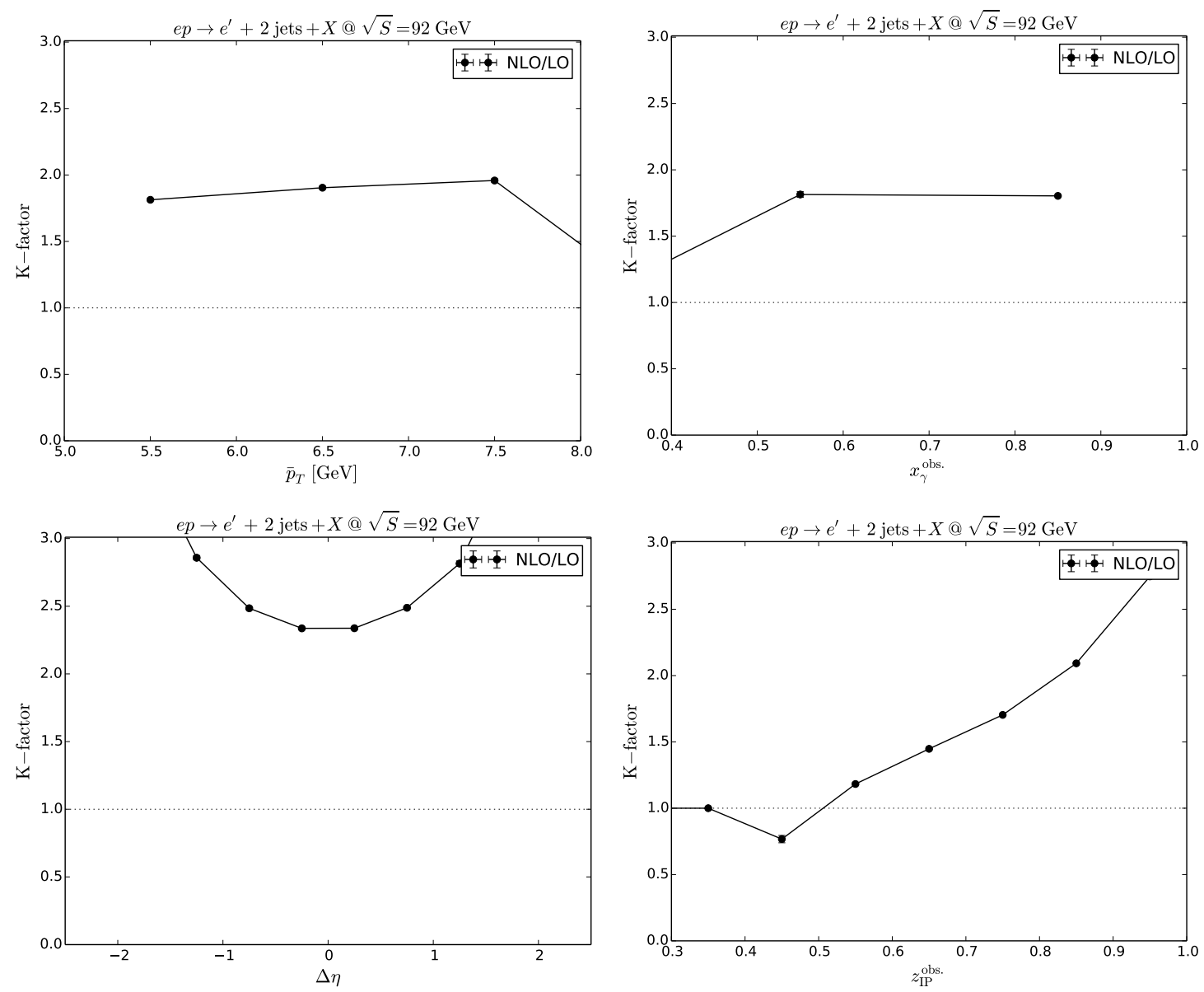

Figure 3. $K$-factors for LO cross sections as a function of the jet average transverse momentum (top left), jet rapidity difference (bottom left) and observed longitudinal momentum fractions in the photon (top right) and pomeron (bottom right).

jet average transverse momentum (top left) extends only to $8 \mathrm{GeV}$, while at HERA with its larger CMS energy of $300-320 \mathrm{GeV}$ it extended to about $15 \mathrm{GeV}$. Consequently, the total cross sections (full black curves) are dominated by contributions from direct photons (dotted green curves) and point-like quark-antiquark pairs, as one can also see from the accessible range in $x_{\gamma}^{\text {obs. }}>0.5$ (top right). It will thus not be easy at this CMS energy to distinguish global factorization breaking from a breaking in only the resolved-photon contribution. Due to the limited available energy, the cross section also requires the largest longitudinal momentum fraction allowed by the kinematic cut of the proton to the pomeron (bottom left) and is dominated by large momentum fractions of the partons (mostly gluons, dashed blue curves) in the pomeron (bottom right).

\section{$3.2 \quad K$-factors}

For detailed event studies with Monte Carlo generators in leading order (LO) QCD, it is useful to know the impact of the NLO corrections. Therefore we show in figure 2 the $K$-factors, i.e. the ratio of NLO over LO cross sections, as a function of jet average trans- 


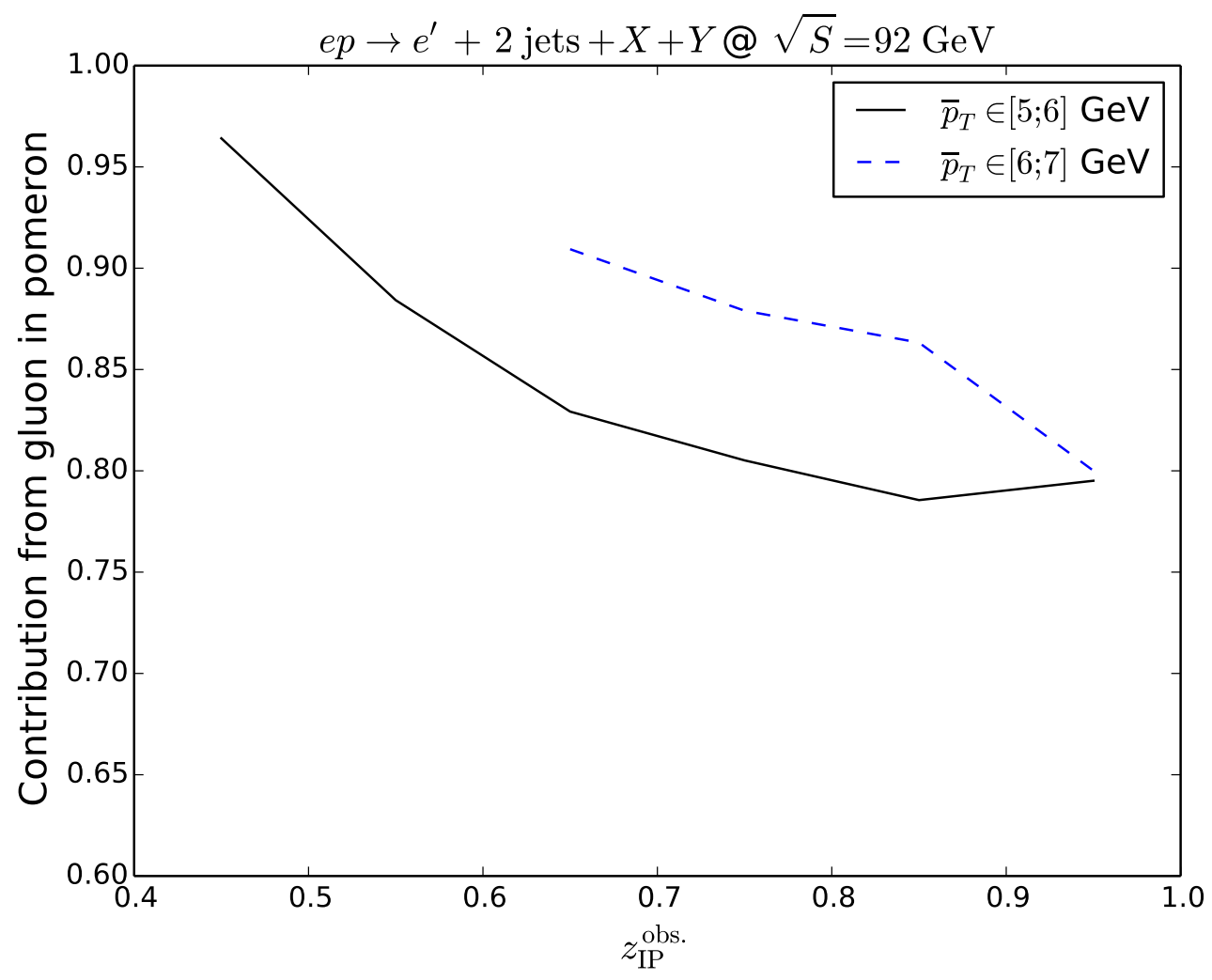

Figure 4. Evolution of the contribution from gluons in the pomeron with the energy scale, set by the jet average transverse momentum, as a function of the longitudinal momentum fraction in the pomeron.

verse momentum (top left), jet rapidity difference (bottom left) and observed longitudinal momentum fractions in the photon (top right) and pomeron (bottom right). At these low scales, the NLO corrections amount to about a factor of two and are thus sizable. At the kinematic edges, i.e. for large rapidity differences $\Delta \eta=\eta_{1}-\eta_{2}$ or values of $z_{\mathbb{P}}^{\text {obs. }}$, they become even larger. For inclusive jet photoproduction at HERA, the corrections at approximate next-to-next-to-leading order (aNNLO) increase the cross section for $p_{T}=20 \mathrm{GeV}$ by another $12 \%$, improving the description of the considered ZEUS data [47]. However, at the same time the scale uncertainty is considerably reduced [19, 20]. This demonstrates that the perturbative expansion remains reliable despite seemingly large $K$-factors at NLO.

\subsection{Evolution of gluon in pomeron contribution}

Although the range in $p_{T}$ is limited at $\sqrt{S}=92 \mathrm{GeV}$, it would be nice to observe the evolution of the diffractive PDFs in the pomeron with the energy scale, set here by the average jet transverse momentum. We therefore compare in figure 4 the fractional contribution of the gluon in the pomeron in the lowest $\bar{p}_{T}$ bin from 5 to $6 \mathrm{GeV}$ to the one in the next-highest bin from 6 to $7 \mathrm{GeV}$. Clearly, this restricts the range in $z_{\mathbb{P}}^{\text {obs. }}$ from above 0.4 to even larger values above 0.6. It also increases the gluon contribution in the pomeron at these large momentum fractions. This corresponds well with the scale evolution and 

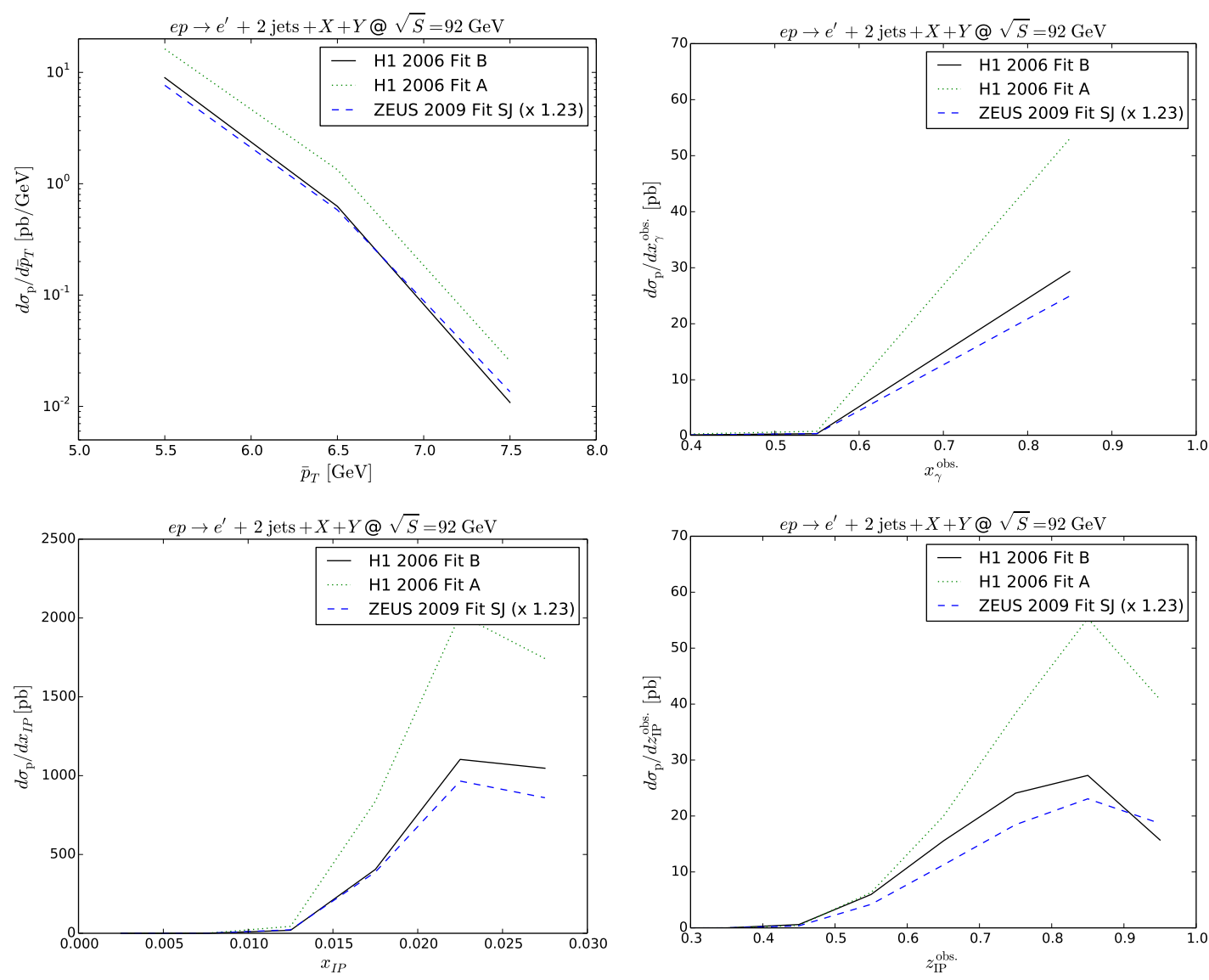

Figure 5. Same as Figure 2, but comparing our NLO QCD predictions using three different sets of diffractive PDFs: H1 2006 Fit B (full black), Fit A (dotted green), and ZEUS 2009 Fit SJ (dashed blue curves). Since the latter have been determined from leading protons, i.e. without dissociation contributions, they must be rescaled by a factor of 1.23 .

relative increase of the rather constant gluon vs. the falling quark singlet density at large $z_{\mathbb{P}}^{\text {obs. }}$, as shown in figure 11 of ref. [5].

\subsection{Dependence on diffractive PDFs}

More important than the evolution of the diffractive PDFs, which should in principle be predictable from perturbative QCD, is the $z_{\mathbb{P}}$ dependence itself, which must be determined from experimental data and which is therefore, despite the considerable progress at HERA, still subject to large uncertainties. In figure 5 we therefore compare our NLO QCD predictions for the EIC using three different fits of the pomeron PDFs to diffractive DIS at HERA: our standard prediction with the frequently used H1 2006 Fit B (full black), the accompanying Fit A (dotted green) [5], and ZEUS 2009 Fit SJ (dashed blue curves) [7]. Since the latter has been obtained from leading protons, i.e. without dissociation contributions, the corresponding cross sections must be and have been multiplied by a factor of 1.23. The main differences between H1 2006 Fits A and B are the starting scales $Q_{0}^{2}=1.75 \mathrm{GeV}^{2}$ and $2.5 \mathrm{GeV}^{2}$, respectively, and the gluon parametrization at large $z_{\mathbb{P}}$, which is singular 

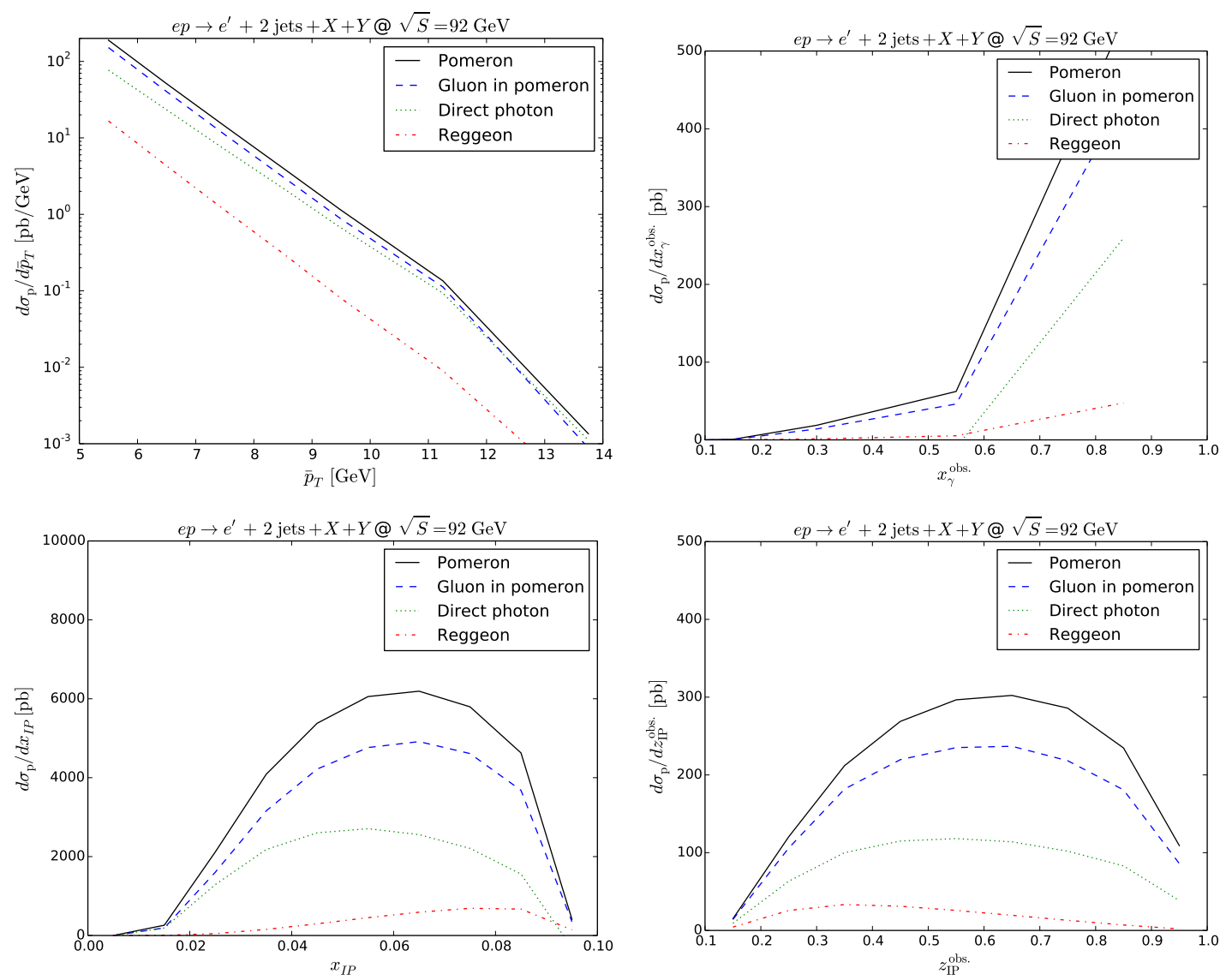

Figure 6. Same as figure 2 , but now with an extended range in $x_{\mathbb{P}}<0.1$. In addition, also the contribution from the subleading reggeon is shown (dot-dashed red curves).

in Fit A and - up to the small- $z_{\mathbb{P}}$ exponential term - constant in Fit B. More precisely, both the gluon and singlet quark densities are parametrized at the starting scale as

$$
z_{\mathbb{P}} f_{i}\left(z_{\mathbb{P}}, Q_{0}^{2}\right)=A_{i} z_{\mathbb{P}}^{B_{i}}\left(1-z_{\mathbb{P}}\right)^{C_{i}}, \quad i=g, q,
$$

where $C_{g}=-0.95 \pm 0.20$ in Fit A and $C_{g}$ is fixed to 0 in Fit B. Attempts have subsequently been made to reduce this uncertainty by adding to the inclusive data also jet production data as in H1 2007 Fit Jets (not used) [6] and ZEUS 2009 Fit SJ [7]. The former uses again $Q_{0}^{2}=2.5 \mathrm{GeV}^{2}$ and results in $C_{g}=0.91 \pm 0.18$, the latter uses $Q_{0}^{2}=1.8 \mathrm{GeV}^{2}$ and results in the smallest uncertainty on $C_{g}=-0.725 \pm 0.082$. Note, however, that $C_{g}$ is intimately linked to the other parameters in the gluon and quark singlet fits, including the pomeron flux factor, so that they cannot be directly compared. What one can observe from figure 5 is that the predictions based on H1 2006 Fit A rise indeed much more steeply as $z_{\mathbb{P}} \rightarrow 1$ and that H1 2006 Fit B, 2007 Fit Jets (not shown) and ZEUS 2009 Fit SJ give comparable results.

\subsection{Range in $x_{\mathbb{P}}$ and reggeon contribution}

The observations of the rather limited range in transverse momentum and the overwhelming importance of the direct photon contribution, that leaves little hope for resolving the 
question of factorization breaking, motivate us to consider also a larger range in $x_{\mathbb{P}}$. In figure 6 we therefore extend it from $x_{\mathbb{P}}<0.03$ to $x_{\mathbb{P}}<0.10$. This immediately enlargens the reach in $\bar{p}_{T}$ from 8 to $14 \mathrm{GeV}$ (top left) and the momentum fraction in the photon from 0.5 down to 0.1 (top right), so that now also resolved photons contribute substantially. Furthermore, the PDFs in the pomeron can now be probed in the entire range of $z_{\mathbb{P}}$ from 0.1 to 1 (bottom right). The increase also seems to be sufficiently large, as the distribution in $x_{\mathbb{P}}$ is no longer peaked at the cut, but around 0.06 (bottom left). This is important since the contribution from the subleading reggeon trajectory increases from less than about $2 \%$ at $x_{\mathbb{P}} \leq 0.03$ to $10-35 \%$ at $x_{\mathbb{P}} \geq 0.06-0.10$. In fact, to obtain a good description of the HERA diffractive DIS data, H1 and ZEUS include an additional sub-leading exchange $(\mathbb{R})$, which has a lower trajectory intercept than the pomeron and which contributes significantly only at large $x_{\mathbb{P}}$ and low $z_{\mathbb{P}}$. This contribution is assumed to factorize in the same way as the pomeron term, such that the diffractive PDFs take the form

$$
f_{i / p}^{D}\left(x, Q^{2}, x_{\mathbb{P}}, t\right)=f_{\mathbb{P} / p}\left(x_{\mathbb{P}}, t\right) \cdot f_{i / \mathbb{P}}\left(z_{\mathbb{P}}, Q^{2}\right)+n_{\mathbb{R}} \cdot f_{\mathbb{R} / p}\left(x_{\mathbb{P}}, t\right) \cdot f_{i / \mathbb{R}}\left(z_{\mathbb{P}}, Q^{2}\right) .
$$

The flux factor $f_{\mathbb{R} / p}$ takes the form of eq. (2.3), normalised via a parameter $A_{\mathbb{R}}$ in the same manner as for the pomeron contribution and with fixed parameters $\alpha_{\mathbb{R}}(0), \alpha_{\mathbb{R}}^{\prime}$ and $B_{\mathbb{R}}$ obtained from other $\mathrm{H} 1$ and ZEUS measurements [5-7]. The parton densities $f_{i / \mathbb{R}}$ of the sub-leading exchange are taken from fits to pion structure function data. We choose the GRV NLO parametrization [48]. Other pion PDFs give similar results [5].

\subsection{EIC beam energy dependence}

Like RHIC, the EIC will accelerate bare charged protons to higher energies per nucleon than it will accelerate nuclei that comprise also neutrons, i.e. not only to $100 \mathrm{GeV}$, but even to $E_{p}=275 \mathrm{GeV}$. At the same time, the most recent plans envisage an electron beam of energy $E_{e}=18 \mathrm{GeV}$ rather than $21 \mathrm{GeV}$ [49]. In figure 7, we therefore repeat our studies for this accelerator design and compare the reach in the different distributions with our default predictions. While a different hadron beam energy will make the extraction of nuclear effects from comparisons of the bare proton baseline with heavy nuclei more difficult, it increases the reach in the kinematic variables relevant for diffraction studies on protons alone. A first example is the reach in average transverse momentum $\bar{p}_{T}$, which is extended from 8 to $12 \mathrm{GeV}$ (top left). Interestingly, an increase in the cut on $x_{\mathbb{P}}$ to 0.1 had a larger effect, extending the reach to $14 \mathrm{GeV}$ (cf. figure 6). A combination of both will therefore

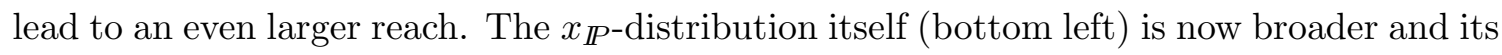
maximum near the cut at 0.0225 less sharp. Similarly to what we observed in the previous section with a larger $x_{\mathbb{P}}$ range, the distributions in longitudinal momentum fraction in the photon (top right) and pomeron (bottom right) also span now larger regions, here from 0.2 (rather than 0.1 ) to 1 . In addition, the corresponding differential cross sections are now larger by one to two orders of magnitude, leading to increased statistics and precision in the corresponding measurements. 

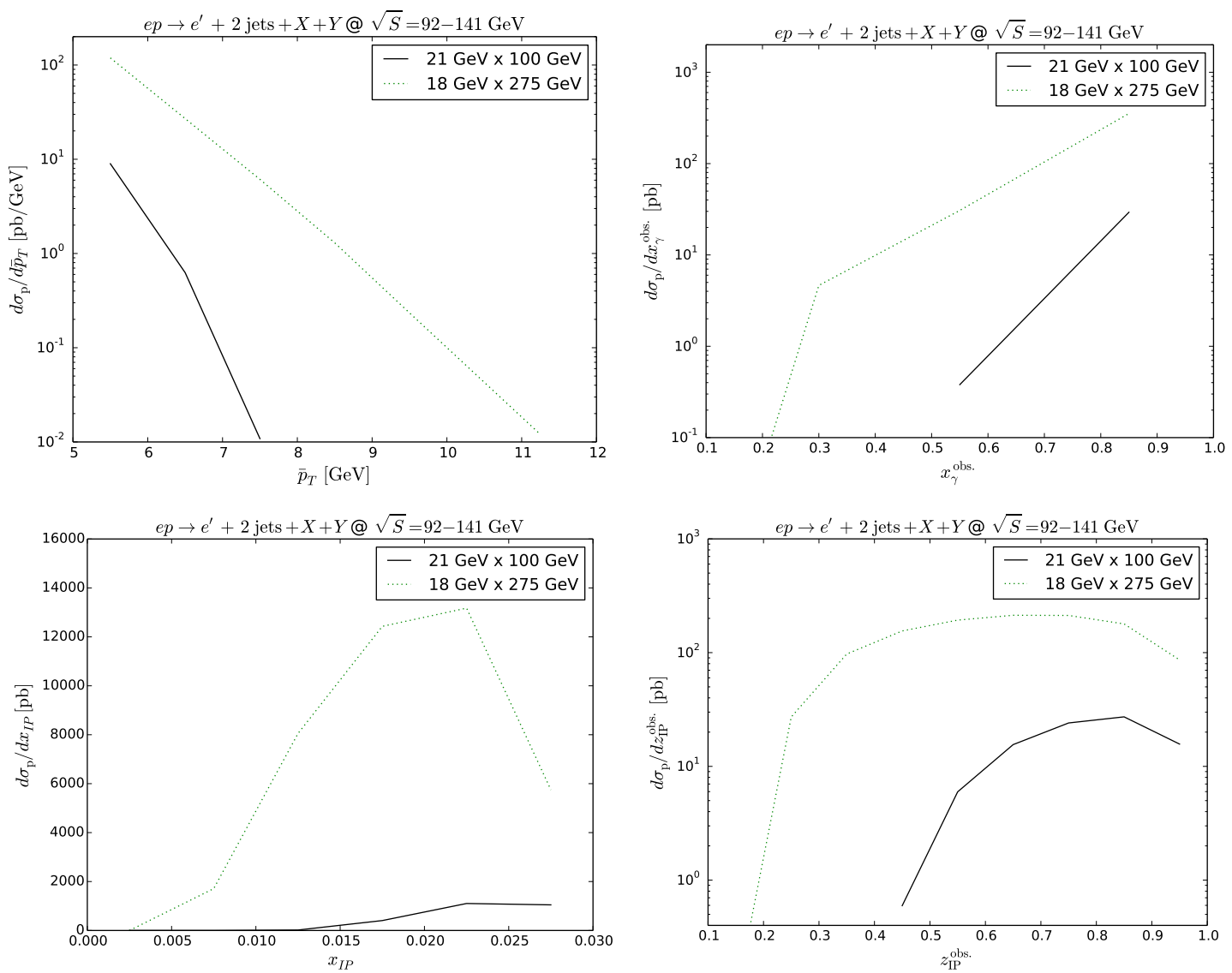

Figure 7. Differential diffractive dijet photoproduction cross sections for collisions of $18 \mathrm{GeV}$ electrons with $275 \mathrm{GeV}$ protons at a CMS energy of $\sqrt{S}=141 \mathrm{GeV}$ (dotted green) compared to our default cross sections for $21 \mathrm{GeV}$ electrons with $100 \mathrm{GeV}$ protons at a CMS of $92 \mathrm{GeV}$ (full black curves).

\subsection{Factorization breaking}

Factorization breaking in diffractive dijet photoproduction is a result of soft inelastic photon interactions with the proton, which populate and thus partially destroy the final-state rapidity gap. This effect is usually described in the literature by a rapidity gap survival factor $S^{2} \leq 1$. Since the magnitude of $S^{2}$ decreases with an increase of the interaction strength between the probe and the target, the pattern of the factorization breaking can be related to various components of the photon [50]. In the laboratory reference frame, the highenergy photon interacts with hadronic targets by fluctuating into various configurations (components) interacting with the target with different cross sections. These fluctuations contain both weakly-interacting (the so-called point-like) components and the components interacting with large cross sections, which are of the order of the vector meson-proton cross sections. This general space-time picture of photon-hadron interactions at high energies is usually realized in the framework of such approaches as the vector meson dominance (VMD) model and its generalizations [51] or the color dipole model [52, 53]. It is also used in the language of collinear factorization, where the photon structure function and 
parton distribution functions (PDFs) are given by a sum of the resolved-photon contribution corresponding to the VMD part of the photon wave function and the point-like (inhomogeneous) term originating from the $\gamma \rightarrow q \bar{q}$ splitting, see, e.g., ref. [44]. Note that the direct-photon contribution to diffractive dijet photoproduction corresponds to the configurations interacting with very small cross sections of the order of $1 / p_{T}^{2}$, which preserves factorization.

The mechanism of factorization breaking in photoproduction is one of the important desiderata of diffraction studies at HERA and could be one of the physics goals of the EIC. The key question is whether factorization still holds for pointlike photons, similarly to DIS, where it has been proven [3], and is only broken for resolved photons [21], similarly to hadron-hadron scattering, where it is known to be broken [8-13], whether it is broken globally to a significant [22] or only a small extent [23], as the H1 and ZEUS data seem to indicate [24], and whether pointlike photon fluctuations into all quark-antiquark pairs [21] or rather those of light quark flavors only [25] rescatter and destroy the rapidity gap. This last point can be related to the mass scheme employed in the photon structure function, i.e. the applicability of dimensional regularization and the zero-mass variable flavor number scheme (ZM-VFNS) vs. the fixed flavor number scheme (FFNS), where the heavy quark mass serves as a regulator. Unfortunately, current photon structure function data do not yet allow to determine the corresponding kinematic ranges. Theory and experience from proton PDFs tells us that the FFNS should be used when $p_{T} \leq m_{q}$, while the ZM-VFNS should be used when $p_{T} \gg m_{q}$. In diffractive photoproduction both at HERA and the EIC, we find ourselves in the transition region $p_{T} \geq m_{q}$, so that (in particular charm) mass effects are indeed still relevant and the charm contribution does not seem to be suppressed.

A precondition for an important contribution from the EIC to settle these questions is a sufficiently large range in direct and resolved photon contributions, i.e. in $x_{\gamma}$. For this reason, we continue to base our numerical studies here on the accelerator design studied in the last subsection with its higher proton beam energy of $E_{p}=275 \mathrm{GeV}$. To avoid the reggeon contribution, which could obscure the situation further, we use the lower cut on $x_{\mathbb{P}}<0.03$. In figure 8 we compare three different schemes of factorization breaking, i.e. a global factorization breaking by a factor of 0.5 as determined in the low- $p_{T}$ measurement of H1 (full black curves) [22], a breaking of the resolved-photon components by a factor of 0.34 as predicted by the two-channel eikonal model (dotted green curves) [14], which is not substantially altered by the finite remainder of collinear quarks and antiquarks [21], and a scheme that interpolates the suppression for photon components of different size as a function of $x_{\gamma}$ [25]. In this last scheme, one expects $S^{2} \approx 0.34$ for the hadron-like component of the photon at small $x_{\gamma}$, and $S^{2} \approx 0.53-0.75$ for the gluon and quark contributions at large $x_{\gamma}$ corresponding to small, but non-negligible factorization breaking due to the point-like component of the resolved photon $[50,54]$. Therefore, we interpolate the effect of factorization breaking with

$$
S_{i}^{2}\left(x_{\gamma}\right) \rightarrow \begin{cases}1, & i=c, \\ A_{q} x_{\gamma}+0.34, & i=u, d, s \\ A_{g} x_{\gamma}+0.34, & i=g\end{cases}
$$



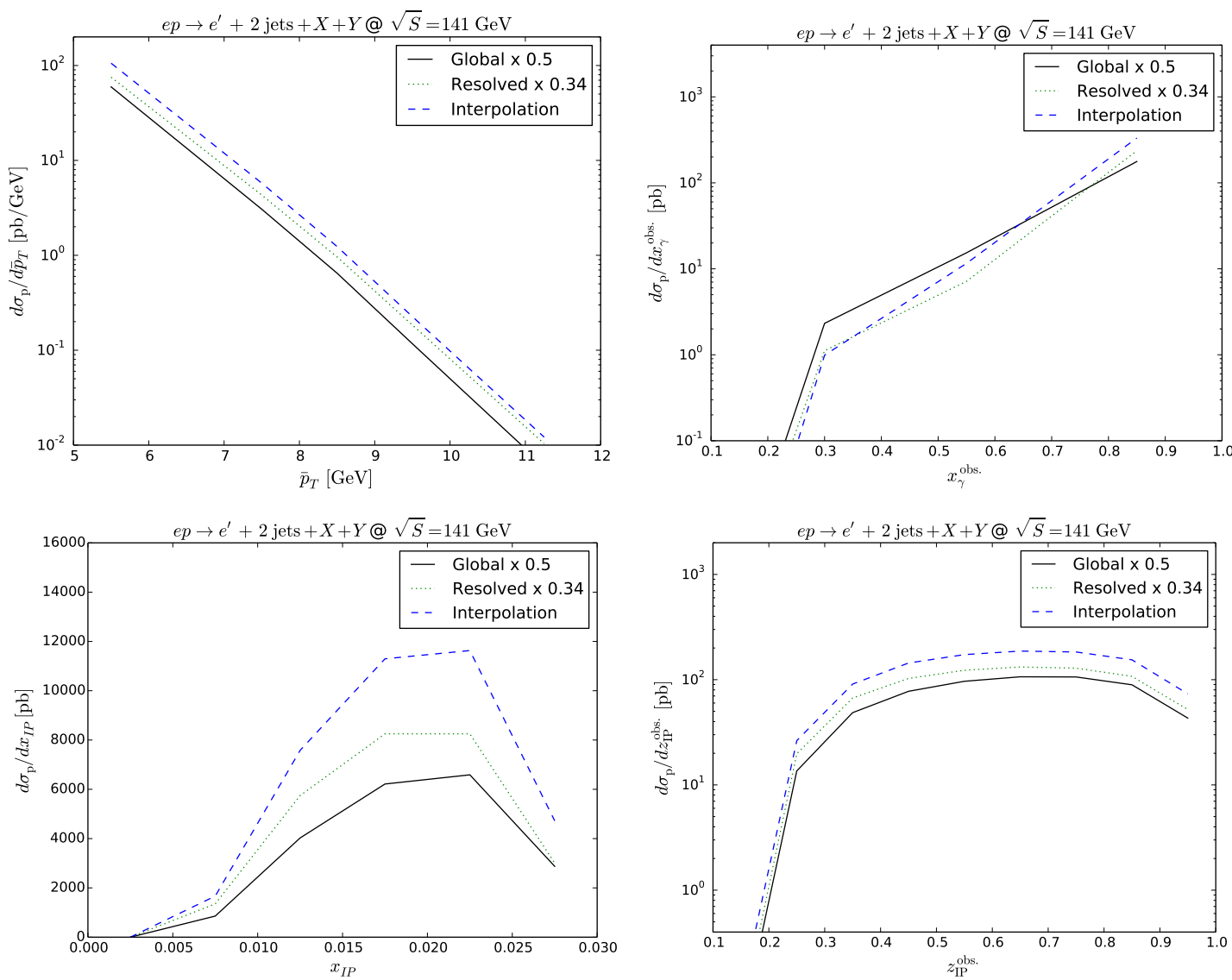

Figure 8. Comparison of factorization breaking schemes in diffractive dijet photoproduction at the EIC for collisions of electrons with energy $18 \mathrm{GeV}$ and protons of energy $275 \mathrm{GeV}$. Shown are NLO QCD predictions with a global suppression by a factor of 0.5 (full black), suppression of resolvedphoton contributions only by a factor of 0.34 (dotted green) and of an interpolated suppression factor that depends on the type of parton in the photon and $x_{\gamma}$ (see text, dashed blue curves).

where $i$ is the parton flavor, $A_{q}=0.37$ and $A_{g}=0.19$ for a hard resolution scale of $p_{T}=5 \mathrm{GeV}$. Note that the model of eq. (3.3) assumes no factorization breaking in the charm quark channel since NLO QCD describes well diffractive photoproduction of open charm in $e p$ scattering (see above).

As one can see from figure 8 (top right), the resolved-only and interpolated schemes

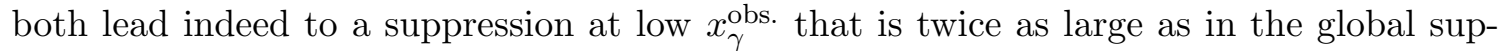
pression scheme. At intermediate values of $x_{\gamma}^{\text {obs. }}$, the interpolated scheme is instead similar to global suppression, while for the pointlike region it is again similar to the resolved-only scheme. Since the distributions fall by two orders of magnitude from $x_{\gamma}^{\text {obs. }}=0.85$ to 0.3 , the differential cross section must be represented on a logarithmic scale and measurements at the EIC will require a high level of precision to distinguish between the different schemes. This should indeed be possible with the planned luminosities up two orders of magnitude larger than at HERA [49]. The shape of the $\bar{p}_{T}$ distribution is also known to be sensitive to different schemes of factorization breaking [24], and this is also true for the global and 
resolved-only schemes at the EIC (figure 8, top left). Interestingly, the interpolation scheme described above differs from the global scheme mostly in the larger normalization, which can be attributed to the fact that the cross section remains dominated by direct photons in the entire $\bar{p}_{T}$ range. As expected, the distributions describing the momentum transfers from the proton to the pomeron (bottom left) and from the pomeron to the hard process (bottom right) have similar shapes for all three suppression schemes and differ again only in normalization.

\section{Diffraction on heavy nuclei}

In the collider mode, it is rather straightforward to measure coherent diffraction on nuclei by selecting events with a large rapidity gap and requiring that no neutrons are produced in the zero-angle calorimeter (ZDC). Practically all events satisfying these requirements would correspond to coherent diffraction. However, measurements of the $t$-dependence would require the use of Roman pots at unrealistically small distances from the beam [30].

\subsection{Nuclear diffractive PDFs and nuclear shadowing}

Nuclear diffractive PDFs are defined similarly to those for nucleons as matrix elements of well-defined quark and gluon operators between nuclear states with the condition that the final-state nucleus does not break, carries longitudinal momentum fraction $1-x_{\mathbb{P}}$, and that the four-momentum transfer squared is $t$.

As in the case of usual nuclear PDFs, nuclear diffractive PDFs are subject to nuclear modifications. In particular, at small $x$ nuclear diffractive PDFs are expected to be suppressed compared to the coherent sum of free nucleon diffractive PDFs due to nuclear shadowing. In the model of leading twist nuclear shadowing [30], nuclear diffractive PDFs $f_{i / A}^{D}$ are obtained by summing a series corresponding to coherent diffractive scattering on one, two $, \ldots, A$ nucleons of the nuclear target, which gives in the small- $x_{\mathbb{P}}$ limit

$$
f_{i / A}^{D}\left(z_{\mathbb{P}}, Q^{2}, x_{\mathbb{P}}\right) \approx 16 \pi B_{\mathrm{diff}} f_{i / p}^{D}\left(z_{\mathbb{P}}, Q^{2}, x_{\mathbb{P}}\right) \int d^{2} \vec{b}\left|\frac{1-e^{-\frac{A}{2}(1-i \eta) \sigma_{\mathrm{soft}}^{i}\left(x, Q^{2}\right) T_{A}(b)}}{(1-i \eta) \sigma_{\mathrm{soft}}^{i}\left(x, Q^{2}\right)}\right|^{2} .
$$

Here $B_{\text {diff }}=6 \mathrm{GeV}^{-2}$ is the slope of the $t$-dependence of the $e p \rightarrow e^{\prime} X p$ differential cross section and $\eta=0.15$ is the ratio of the real to imaginary parts of the corresponding scattering amplitude; $T_{A}(b)=\int d z \rho_{A}(b, z)$ is the optical nuclear density, where $\rho_{A}(b, z)$ is the nuclear density [55] and $b$ is the transverse position (impact parameter) of the interacting nucleon in the nucleus; $\sigma_{\text {soft }}^{i}=30 \mathrm{mb}$ is an effective cross section controlling the strength of the interaction with target nucleons, which can be estimated using models of the hadronic structure of the virtual photon. The used value of $\sigma_{\text {soft }}^{i}$ corresponds to the scenario with the larger nuclear shadowing of ref. [30]. One can see from eq. (4.1) that an account of nuclear shadowing leads in principle to an explicit violation of Regge factorization for nuclear diffractive PDFs.

A numerical analysis of eq. (4.1) shows [30] that the effect of nuclear shadowing in most of the kinematics only weakly depends on flavor $i$, the momentum fractions $z_{\mathbb{P}}$ and 
$x_{\mathbb{P}}$, and the resolution scale $Q^{2}$. Therefore, to a good approximation, one has the following relation

$$
f_{i / A}^{D}\left(z_{\mathbb{P}}, Q^{2}, x_{\mathbb{P}}\right) \approx A R(x, A) f_{i / p}^{D}\left(z_{\mathbb{P}}, Q^{2}, x_{\mathbb{P}}\right),
$$

where $R(x, A) \approx 0.65$ is a weak function of $x$ and $A$ and is calculated using eq. (4.1).

\subsection{NLO QCD predictions for the EIC}

Our predictions for the NLO QCD cross sections for coherent diffractive dijet photoproduction in $e A \rightarrow e^{\prime}+2$ jets $+X+A$ scattering with different nuclear beams (U-238, Au-197, $\mathrm{Cu}-63$, and C-12) at the EIC in our default set-up with $\sqrt{S}=92 \mathrm{GeV}$ are shown in figure 9. The cross sections are shown as functions of the jet average transverse momentum (top left), the jet rapidity difference (bottom left), the observed longitudinal momentum fractions of partons in the photon (top right) and pomeron (bottom right). As naturally follows from eq. (4.2), the shapes of the nuclear cross sections repeat those for the proton shown in figure 2. The free proton diffractive PDFs as parameterized in H1 2006 Fit $\mathrm{B}$ [5] have been divided by a factor of 1.23 in order to take into account the fact that here we have no diffractive dissociation contributions, as the heavy nucleus is assumed to stay intact and no neutrons are assumed to be produced in the ZDC.

\subsection{Factorization breaking}

As discussed in section 3.7, collinear QCD factorization is violated in diffractive dijet photoproduction due to soft inelastic interactions with the hadronic target. While the mechanism of this factorization breaking is not yet established, it is natural to expect that the effect will be more pronounced for nuclear targets since the gap survival probability is significantly smaller for nuclei than for the proton. For instance, using the commonly used two-state eikonal model [12-14], one estimates [28] that the suppression factor in the relevant energy range is $S^{2}=0.4$ for the proton and $S^{2}=0.04$ for heavy nuclei.

Figure 10 shows our predictions for the NLO QCD cross section for coherent diffractive dijet photoproduction with Au-197 beams at the EIC. For heavy nuclei, we unfortunately cannot enhance the resolved-photon contribution to increase the differences between competing factorization breaking schemes by assuming a higher beam energy of $275 \mathrm{GeV}$ as for protons, but we remain limited to $100 \mathrm{GeV}$ per nucleon. We can, however, perform our study for the larger range in $x_{\mathbb{P}}<0.10$ instead of 0.03 , which, as we have seen in figure 6 , also increases the accessible range in $x_{\gamma}$. In figure 10, the solid lines correspond to the case where we apply a global suppression factor of $S^{2}=0.5$ to our calculated cross sections as in the proton case, while the dotted lines correspond to the factorization breaking scenario, where only the resolved-photon contribution is suppressed, now by a factor of $S^{2}=0.04$ (see the discussion above). A comparison of these two schemes shows that due to the dominance of the direct photon contribution in most of the EIC kinematics, they lead to similar shapes of the kinematic distributions that differ only in normalization, with the important exception of the $x_{\gamma}$-distribution (top right), which below values of 0.5 is negligible for resolved-only suppression and has potentially measurable support only in the global suppression scheme. 

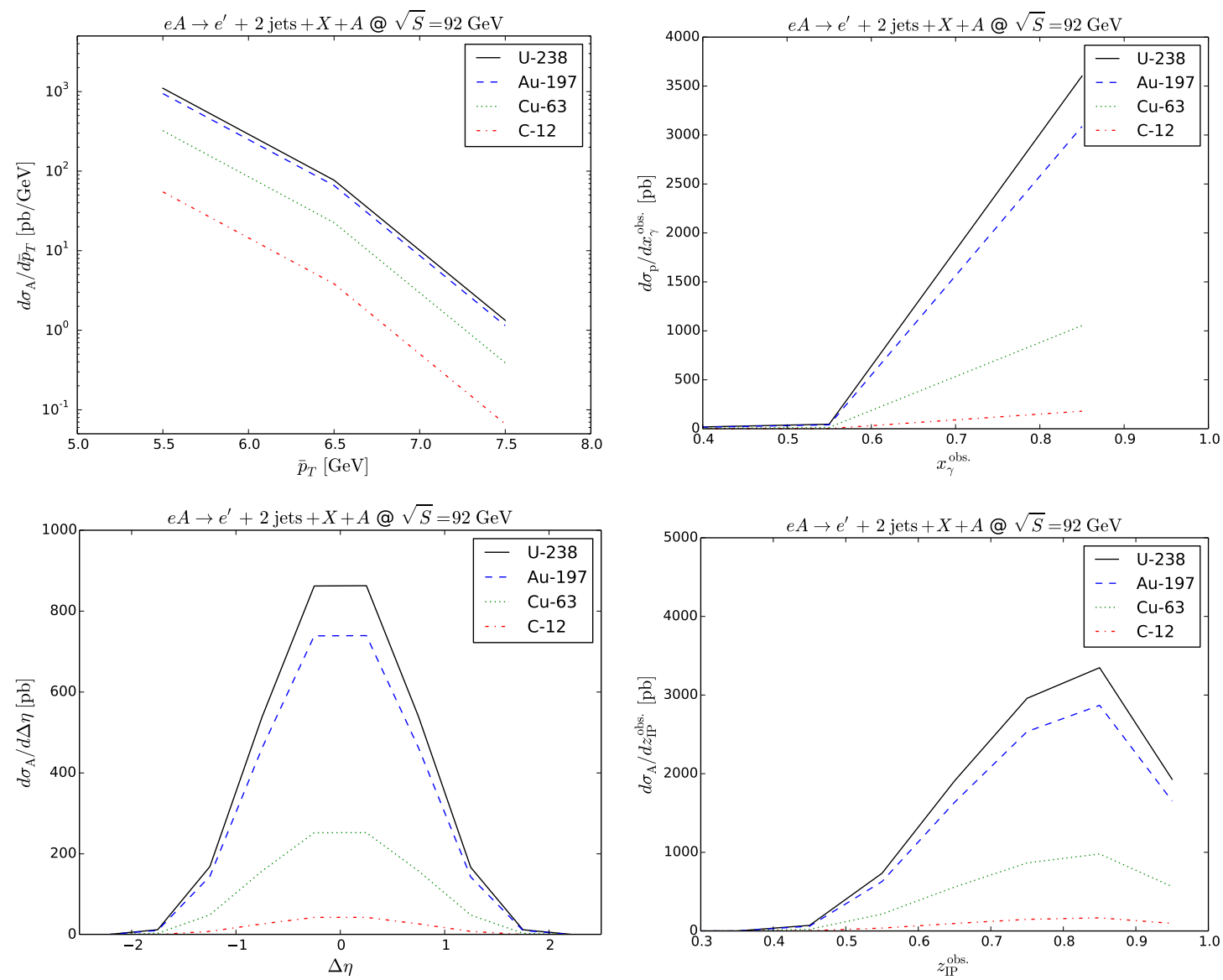

Figure 9. NLO QCD cross sections for coherent diffractive dijet photoproduction $e A \rightarrow e^{\prime}+2$ jets + $X+A$ with various nuclear beams and a center-of-mass energy per nucleon of $\sqrt{S}=92 \mathrm{GeV}$ at the EIC. The cross sections are shown as functions of the jet average transverse momentum (top left) and rapidity difference (bottom left) as well as the longitudinal momentum fractions in the photon (top right) and pomeron (bottom right).

\section{Conclusion}

To summarize, we have in this paper presented a first and extensive study of diffractive dijet photoproduction at the recently approved EIC. Using our established formalism of NLO QCD calculations, we have illuminated various aspects of this interesting scattering process. We started by determining the cross sections to be expected in the most important differential distributions as well as the size of the NLO corrections. We then discussed the sensitivity to pomeron PDFs as a function of momentum fraction and resolution scale as well as the contribution from the higher reggeon tractectory. One of our two main results is that the EIC has the potential to address conclusively the mechanism of factorization breaking, but that this will require a high proton beam energy and/or a large longitudinal momentum transfer from the proton/nucleus to the pomeron. Then the question will hopefully be answered whether and to what extent factorization breaking occurs globally in photoproduction or whether only the resolved photon contribution or some of its compo- 

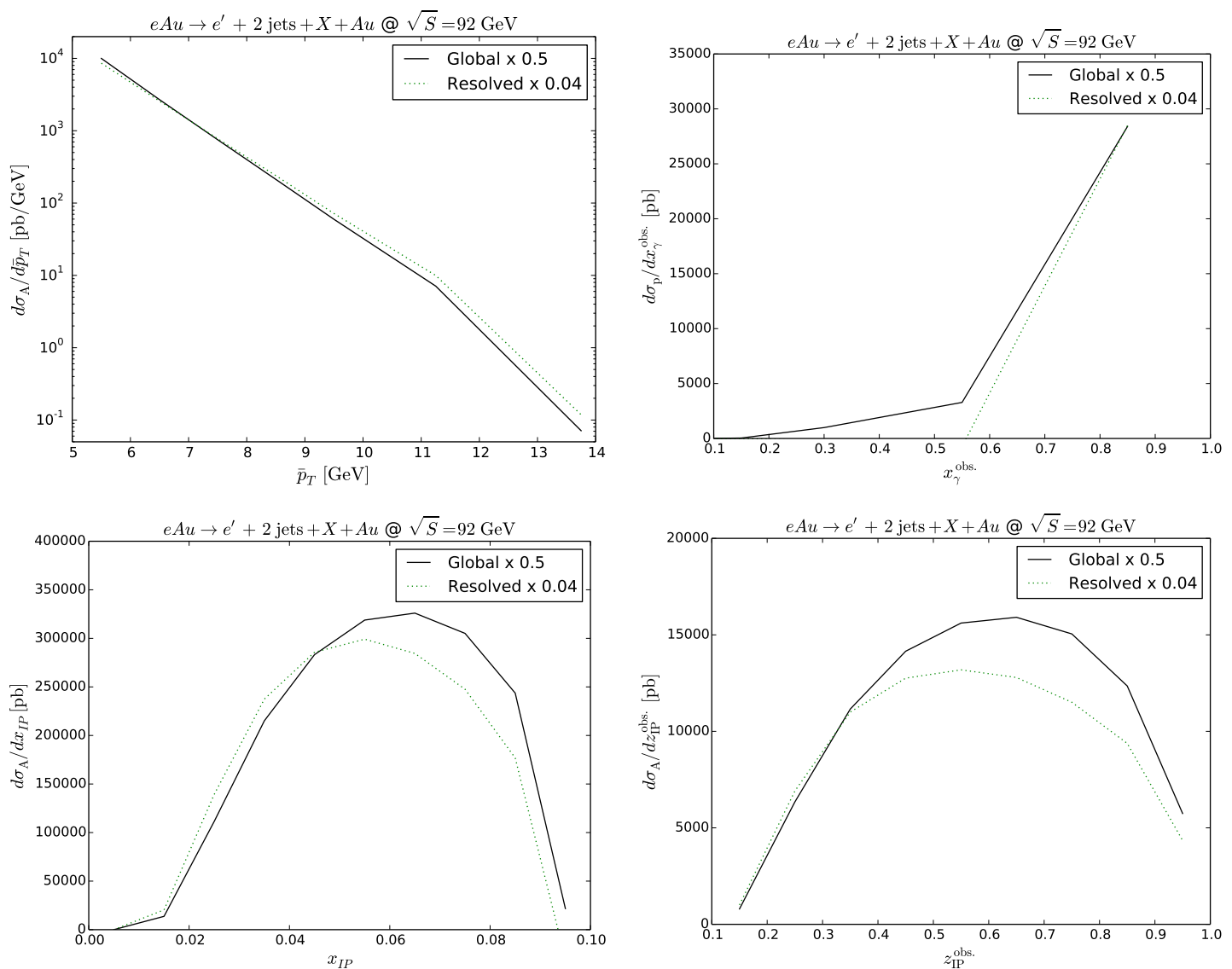

Figure 10. NLO QCD cross sections for coherent diffractive dijet photoproduction $e A u \rightarrow e^{\prime}+$ 2 jets $+X+A u$ at $\sqrt{S}=92 \mathrm{GeV}$ at the EIC with an extended range in $x_{\mathbb{P}}<0.1$. Shown are distributions in the jet average transverse momentum (top left) as well as the (observed) longitudinal momentum fractions of the photon (top right), the pomeron (bottom left) and the partons in the pomeron (bottom right). We compare two different schemes of factorization breaking, i.e. global suppression by a factor of 0.5 (full black) with only resolved-photon suppression by a factor of 0.04 (dotted green curves).

nents (light/heavy quark-antiquark pairs, VMD contributions) are suppressed. Our second main result comprises predictions for diffractive dijet photoproduction on nuclei, which might - perhaps for the first time - give access to nuclear diffractive PDFs. Here, we made numerical predictions for four different nuclei, ranging from carbon to uranium, as well as again for different factorization breaking schemes.

As an outlook, let us point out that at HERA also dijet production with leading neutrons has been studied [56-58]. These processes have been interpreted in terms of virtual charged-pion exchanges and gave first information on the structure of (virtual) pions at previously unaccessible values of $x[59,60]$. It would be very interesting to continue these studies at the EIC, also in view of possible factorization breaking in these processes [61], and perhaps even extend them to dissociation processes in collisions with heavy nuclei. 


\section{Acknowledgments}

The authors thank Wim Cosyn and the other organizers of the 1st EIC Yellow Report workshop at Temple University for stimulating this study and Paul Newman for his interesting questions, in particular on the impact of a larger range in $x_{\mathbb{P}}$. Financial support by the DFG through the grant KL 1266/9-1 within the framework of the joint GermanRussian project "New constraints on nuclear parton distribution functions at small $x$ from dijet production in $\gamma A$ collisions at the LHC" is gratefully acknowledged. VG's research is also supported in part by RFBR, research project 17-52-12070.

Open Access. This article is distributed under the terms of the Creative Commons Attribution License (CC-BY 4.0), which permits any use, distribution and reproduction in any medium, provided the original author(s) and source are credited.

\section{References}

[1] P. Newman and M. Wing, The hadronic final state at HERA, Rev. Mod. Phys. 86 (2014) 1037 [arXiv: 1308. 3368] [INSPIRE].

[2] M. Klasen, Theory of hard photoproduction, Rev. Mod. Phys. 74 (2002) 1221 [hep-ph/0206169] [INSPIRE].

[3] J.C. Collins, Proof of factorization for diffractive hard scattering, Phys. Rev. D 57 (1998) 3051 [Erratum ibid. D 61 (2000) 019902] [hep-ph/9709499] [INSPIRE].

[4] G. Ingelman and P.E. Schlein, Jet structure in high mass diffractive scattering, Phys. Lett. B $152(1985) 256$.

[5] H1 collaboration, Measurement and QCD analysis of the diffractive deep-inelastic scattering cross-section at HERA, Eur. Phys. J. C 48 (2006) 715 [hep-ex/0606004] [InSPIRE].

[6] H1 collaboration, Dijet cross sections and parton densities in diffractive DIS at HERA, JHEP 10 (2007) 042 [arXiv:0708.3217] [INSPIRE].

[7] ZEUS collaboration, A QCD analysis of ZEUS diffractive data, Nucl. Phys. B 831 (2010) 1 [arXiv: 0911.4119] [INSPIRE].

[8] CDF collaboration, Diffractive dijets with a leading antiproton in $\bar{p} p$ collisions at $\sqrt{s}=1800$ GeV, Phys. Rev. Lett. 84 (2000) 5043 [inSPIRE].

[9] CDF collaboration, Diffractive dijet production at $\sqrt{s}=630 \mathrm{GeV}$ and $1800 \mathrm{GeV}$ at the Fermilab Tevatron, Phys. Rev. Lett. 88 (2002) 151802 [hep-ex/0109025] [INSPIRE].

[10] CMS, TOTEM collaboration, Measurement of single-diffractive dijet production in proton-proton collisions at $\sqrt{s}=8 \mathrm{TeV}$ with the CMS and TOTEM experiments, arXiv:2002.12146 [INSPIRE].

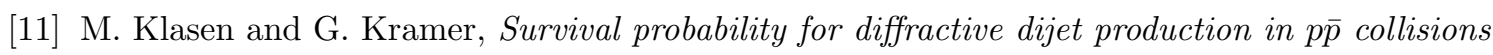
from next-to-leading order calculations, Phys. Rev. D 80 (2009) 074006 [arXiv:0908.2531] [INSPIRE].

[12] V.A. Khoze, A.D. Martin and M.G. Ryskin, Soft diffraction and the elastic slope at Tevatron and LHC energies: a MultiPomeron approach, Eur. Phys. J. C 18 (2000) 167 [hep-ph/0007359] [INSPIRE]. 
[13] A.B. Kaidalov, V.A. Khoze, A.D. Martin and M.G. Ryskin, Probabilities of rapidity gaps in high-energy interactions, Eur. Phys. J. C 21 (2001) 521 [hep-ph/0105145] [INSPIRE].

[14] A.B. Kaidalov, V.A. Khoze, A.D. Martin and M.G. Ryskin, Unitarity effects in hard diffraction at HERA, Phys. Lett. B 567 (2003) 61 [hep-ph/0306134] [INSPIRE].

[15] M. Klasen, G. Kramer and S.G. Salesch, Photoproduction of jets at HERA: Comparison of next-to-leading order calculation with ZEUS data, Z. Phys. C 68 (1995) 113 [INSPIRE].

[16] M. Klasen and G. Kramer, Inclusive dijet production at HERA: direct photon cross-sections in next-to-leading order QCD, Z. Phys. C 72 (1996) 107 [hep-ph/9511405] [InSPIRE].

[17] M. Klasen and G. Kramer, Inclusive two jet production at HERA: direct and resolved cross-sections in next-to-leading order QCD, Z. Phys. C 76 (1997) 67 [hep-ph/9611450] [INSPIRE].

[18] M. Klasen, T. Kleinwort and G. Kramer, Inclusive jet production in $\gamma p$ and $\gamma \gamma$ processes: direct and resolved photon cross sections in next-to-leading order QCD, Eur. Phys. J. direct 1 (1998) 1 [hep-ph/9712256] [INSPIRE].

[19] M. Klasen, G. Kramer and M. Michael, Next-to-next-to-leading order contributions to jet photoproduction and determination of $\alpha_{s}$, Phys. Rev. D 89 (2014) 074032 [arXiv:1310.1724] [INSPIRE].

[20] T. Biekötter, M. Klasen and G. Kramer, Next-to-next-to-leading order contributions to inclusive jet production in deep-inelastic scattering and determination of $\alpha_{s}$, Phys. Rev. D 92 (2015) 074037 [arXiv: 1508.07153] [INSPIRE].

[21] M. Klasen and G. Kramer, Factorization scheme and scale dependence in diffractive dijet production at low $Q^{2}$, J. Phys. G 31 (2005) 1391 [hep-ph/0506121] [INSPIRE].

[22] H1 collaboration, Tests of QCD factorisation in the diffractive production of dijets in deep-inelastic scattering and photoproduction at HERA, Eur. Phys. J. C 51 (2007) 549 [hep-ex/0703022] [INSPIRE].

[23] ZEUS collaboration, Diffractive photoproduction of dijets in ep collisions at HERA, Eur. Phys. J. C 55 (2008) 177 [arXiv:0710.1498] [INSPIRE].

[24] M. Klasen and G. Kramer, Review of factorization breaking in diffractive photoproduction of dijets, Mod. Phys. Lett. A 23 (2008) 1885 [arXiv:0806.2269] [InSPIRE].

[25] V. Guzey and M. Klasen, A fresh look at factorization breaking in diffractive photoproduction of dijets at HERA at next-to-leading order QCD, Eur. Phys. J. C 76 (2016) 467 [arXiv: 1606. 01350] [INSPIRE].

[26] A.J. Baltz, The physics of ultraperipheral collisions at the LHC, Phys. Rept. 458 (2008) 1 [arXiv:0706.3356] [INSPIRE].

[27] V. Guzey and M. Klasen, Inclusive dijet photoproduction in ultraperipheral heavy ion collisions at the CERN Large Hadron Collider in next-to-leading order QCD, Phys. Rev. C 99 (2019) 065202 [arXiv: 1811.10236] [INSPIRE].

[28] V. Guzey and M. Klasen, Diffractive dijet photoproduction in ultraperipheral collisions at the LHC in next-to-leading order QCD, JHEP 04 (2016) 158 [arXiv: 1603.06055] [INSPIRE].

[29] V. Guzey and M. Klasen, Constraints on nuclear parton distributions from dijet photoproduction at the LHC, Eur. Phys. J. C 79 (2019) 396 [arXiv:1902.05126] [inSPIRE]. 
[30] L. Frankfurt, V. Guzey and M. Strikman, Leading twist nuclear shadowing phenomena in hard processes with nuclei, Phys. Rept. 512 (2012) 255 [arXiv:1106.2091] [INSPIRE].

[31] A. Accardi et al., Electron ion collider: the next QCD frontier, Eur. Phys. J. A 52 (2016) 268 [arXiv: 1212.1701] [INSPIRE].

[32] D. Boer, P.J. Mulders, C. Pisano and J. Zhou, Asymmetries in heavy quark pair and dijet production at an EIC, JHEP 08 (2016) 001 [arXiv:1605.07934] [INSPIRE].

[33] M. Klasen, K. Kovarik and J. Potthoff, Nuclear parton density functions from jet production in DIS at an EIC, Phys. Rev. D 95 (2017) 094013 [arXiv:1703. 02864] [INSPIRE].

[34] R. Boughezal, F. Petriello and H. Xing, Inclusive jet production as a probe of polarized parton distribution functions at a future EIC, Phys. Rev. D 98 (2018) 054031 [arXiv: 1806.07311] [INSPIRE].

[35] X. Liu, F. Ringer, W. Vogelsang and F. Yuan, Lepton-jet correlations in deep inelastic scattering at the electron-ion collider, Phys. Rev. Lett. 122 (2019) 192003 [arXiv: 1812.08077] [INSPIRE].

[36] M. Arratia, Y. Song, F. Ringer and B. Jacak, Jets as precision probes in electron-nucleus collisions at the electron-ion collider, arXiv:1912.05931 [INSPIRE].

[37] Y. Hatta, N. Mueller, T. Ueda and F. Yuan, QCD resummation in hard diffractive dijet production at the electron-ion collider, Phys. Lett. B 802 (2020) 135211 [arXiv:1907.09491].

[38] H. Mäntysaari, N. Mueller, F. Salazar and B. Schenke, Multi-gluon correlations and evidence of saturation from dijet measurements at an electron ion collider, Phys. Rev. Lett. 124 (2020) 112301 [arXiv:1912.05586] [INSPIRE].

[39] M. Klasen and K. Kovařík, Nuclear parton density functions from dijet photoproduction at the EIC, Phys. Rev. D 97 (2018) 114013 [arXiv:1803.10985] [INSPIRE].

[40] V. Guzey and M. Klasen, NLO QCD predictions for dijet photoproduction in lepton-nucleus scattering at the EIC, LHeC, HE-LHeC and FCC, arXiv:2003.09129 [INSPIRE].

[41] X. Chu, E.-C. Aschenauer, J.-H. Lee and L. Zheng, Photon structure studied at an electron ion collider, Phys. Rev. D 96 (2017) 074035 [arXiv:1705.08831] [InSPIRE].

[42] E.-C. Aschenauer, K. Lee, B.S. Page and F. Ringer, Jet angularities in photoproduction at the electron-ion collider, Phys. Rev. D 101 (2020) 054028 [arXiv:1910.11460] [INSPIRE].

[43] S. Frixione, M.L. Mangano, P. Nason and G. Ridolfi, Improving the Weizsacker-Williams approximation in electron-proton collisions, Phys. Lett. B 319 (1993) 339 [hep-ph/9310350] [INSPIRE].

[44] M. Gluck, E. Reya and A. Vogt, Photonic parton distributions, Phys. Rev. D 46 (1992) 1973 [INSPIRE].

[45] M. Cacciari, G.P. Salam and G. Soyez, The anti- $k_{t}$ jet clustering algorithm, JHEP 04 (2008) 063 [arXiv: 0802.1189] [INSPIRE].

[46] M. Klasen and G. Kramer, Dijet cross-sections at $o\left(\alpha \alpha_{S}^{2}\right)$ in photon-proton collisions, Phys. Lett. B 366 (1996) 385 [hep-ph/9508337] [INSPIRE].

[47] ZEUS collaboration, Inclusive-jet photoproduction at HERA and determination of alphas, Nucl. Phys. B 864 (2012) 1 [arXiv: 1205.6153] [InSPIRE].

[48] M. Gluck, E. Reya and A. Vogt, Pionic parton distributions, Z. Phys. C 53 (1992) 651 [INSPIRE]. 
[49] J. Skaritka et al., Conceptual design of a polarized electron ion collider at Brookhaven National Laboratory, PoS (PSTP2017) 015.

[50] A.B. Kaidalov, V.A. Khoze, A.D. Martin and M.G. Ryskin, Factorization breaking in diffractive dijet photoproduction at HERA, Eur. Phys. J. C 66 (2010) 373 [arXiv:0911.3716] [INSPIRE].

[51] T.H. Bauer, R.D. Spital, D.R. Yennie and F.M. Pipkin, The hadronic properties of the photon in high-energy interactions, Rev. Mod. Phys. 50 (1978) 261 [Erratum ibid. 51 (1979) 407] [INSPIRE].

[52] J. Nemchik, N.N. Nikolaev, E. Predazzi and B.G. Zakharov, Color dipole systematics of diffractive photoproduction and electroproduction of vector mesons, Phys. Lett. B 374 (1996) 199 [hep-ph/9604419] [INSPIRE].

[53] H. Kowalski, L. Motyka and G. Watt, Exclusive diffractive processes at HERA within the dipole picture, Phys. Rev. D 74 (2006) 074016 [hep-ph/0606272] [INSPIRE].

[54] M. Klasen and G. Kramer, Suppression factors in diffractive photoproduction of dijets, Eur. Phys. J. C 70 (2010) 91 [arXiv:1006.4964] [INSPIRE].

[55] H. De Vries, C.W. De Jager and C. De Vries, Nuclear charge and magnetization density distribution parameters from elastic electron scattering, Atom. Data Nucl. Data Tabl. $\mathbf{3 6}$ (1987) 495.

[56] H1 collaboration, Measurement of dijet cross sections in ep interactions with a leading neutron at HERA, Eur. Phys. J. C 41 (2005) 273 [hep-ex/0501074] [INSPIRE].

[57] ZEUS collaboration, Measurement of dijet cross-sections for events with a leading neutron in photoproduction at HERA, Nucl. Phys. B 596 (2001) 3 [hep-ex/0010019] [INSPIRE].

[58] ZEUS collaboration, Measurement of dijet photoproduction for events with a leading neutron at HERA, Nucl. Phys. B 827 (2010) 1 [arXiv:0909.3032] [InSPIRE].

[59] M. Klasen and G. Kramer, Photoproduction of jets on a virtual pion target in next-to-leading order QCD, Phys. Lett. B 508 (2001) 259 [hep-ph/0103056] [INSPIRE].

[60] M. Klasen, The Pion structure function and jet production in $\gamma p \rightarrow n X, J$. Phys. G 28 (2002) 1091 [hep-ph/0107011] [INSPIRE].

[61] M. Klasen and G. Kramer, Factorization breaking in dijet photoproduction with a leading neutron, Eur. Phys. J. C 49 (2007) 957 [hep-ph/0608235] [INSPIRE]. 